\title{
Las regiones de montañas como espacios de oportunidad para la integración bioceánica: el ejemplo del paso de Jama en los Andes Centrales ${ }^{1}$
}

\author{
Sigrun Kanitscheider ${ }^{2}$ y Oliver Bender ${ }^{3}$
}

\begin{abstract}
RESUMEN
En el debate político la mejora de la infraestructura de transporte en la Cordillera de Los Andes es visto como un factor clave para el desarrollo económico de las regiones periféricas. Tomando el caso de la carretera internacional recientemente ampliada que atraviesa los Andes Centrales, entre Argentina y Chile, sobre la base de estadísticas oficiales es posible identificar un importante flujo de tráfico generado a escala regional. Sin embargo, el potencial desarrollo de un enlace de transporte internacional a través de una zona de alta montaña no se puede evaluar, exclusivamente, sobre la justificación económica de la carretera. Numerosos factores también entran en juego como son los políticos, jurídico y étnico-histórico. En esta investigación se exploran estos factores en caso del paso de Jama y se presentan sus cambios y persistencias. Esto permite analizar la importancia económica de la carretera para el desarrollo de la región, así como también para futuras propuestas de construcción de vías por pasos montañosos.
\end{abstract}

Palabras clave: Paso de montaña, integración bioceánica, desarrollo regional, transporte, Jama.

\begin{abstract}
In policy debates, improving the transport infrastructure in the Andes is seen as a key factor for the economic development of peripheral regions. Taking the case of the newly widened trunk road through the Central Andes between Argentina and Chile, based on official statistics I will identify the traffic flows actually generated by the mountain pass road at regional and global scale. However, the development potential of an international transport link through the high mountain area cannot be assessed on the economic justification of the road alone. Many other factors come into play here. They stem from the natural landscape, from political, legal as well as ethnic-historical contexts. I shall explore these factors in the case study and present their persistence vis-a-vis changes. This opens up the possibility of analysing the economic significance of an existing mountain pass road for the development of the region as well as gauging such significance for proposed road construction projects.
\end{abstract}

Key words: Mountain pass road, bioceanic integration, regional development, transport, Jama.

1 Artículo recibido el 31 de marzo de 2012, aceptado el 31 enero de 2013 y corregido el 4 de marzo de 2013.
2 Institute for Interdisciplinary Mountain Research, Austrian Academy of Sciences (Austria). E-mail: sigrun.kanitscheider@oeaw.ac.at

3 Institute for Interdisciplinary Mountain Research, Austrian Academy of Sciences (Austria).

Email: oliver.bender@oeaw.ac.at 
"Jujuy tiene salida" es el lema utilizado por el gobierno de la provincia de Jujuy para promover la carretera sobre el paso de Jama. La selección de la frase revela por un lado la esperanza de la política en el potencial desarrollador del paso que conecta una región periférica de Argentina con los puertos norteños de Chile. Por otro lado, la expresión concede el problema de la situación geopolítica de la provincia en el extremo noroeste de Argentina. En contraposición a muchas regiones en Europa, especialmente en Los Alpes, donde los flujos de tráfico generalmente son considerados nocivos para la calidad de vida en asentamientos humanos (debate del tránsito) (Borsdorf, 2004), en el contexto latinoamericano y particularmente en las regiones periféricas el tráfico es calificado la fuerza motriz de la economía y por lo tanto, del desarrollo regional.

En la discusión académica existe acuerdo sobre la relación entre infraestructura de transporte y desarrollo regional (Vickerman, 1991). Generalmente, el nivel de prosperidad de una región corresponde con la densidad y calidad de su infraestructura viaria. Sin embargo, eso no implica que exista una relación causal entre prosperidad e infraestructura y todavía menos explica la dirección de aqueIla. ¿El desarrollo regional es el resultado de infraestructura vial o es a la inversa? (Banister \& Berechman, 2001: 214). En algunos casos, la inversión en infraestructura viaria incluso puede generar la fuga del potencial de desarrollo endógeno de una región por que la reducción de los costos de transporte fomenta la concentración de actividades económicas (Eckey \& Kosfeld, 2004).

Considerando diversos enfoques de investigación (Krugmann, 1991, Njenga \& Davis, 2003: 219, MacKinnon et al., 2008: 24, Olsson, 2009, Button, 2010: 419-460), es evidente que invertir en infraestructura de transporte puede funcionar como catalizador, es decir las inversiones permiten expansión e innovación económica siempre y cuando se cumplen condiciones necesarias adicionales. Para Wilson (2009) esta es la dinámica económica en la región. Banister \& Berechman (2001) listan un fajo de tres condiciones (el marco económico, inversiones y el marco político/institucional). Todas tienen que ser adecuadas para que semejantes inversiones resulten en el desarrollo económico de la región. Generalmente, los modelos que evalúan los grandes proyectos de infraestructura de transporte antes de su realización, se basan en un análisis de la relación costebeneficio netamente económico (Salling \& Banister, 2009) sin tener en cuenta que la población local no siempre sigue la lógica económica por sus experiencias y tradiciones locales.

Lakshmanan \& Chatterjee (2005) dan mucha importancia al contexto en el cual se realizan las mejoras de infraestructura y enfatizan las grandes escalas de tiempo en las que se efectúan los cambios. Aunque a nivel global la importancia económica de los costos de transporte disminuyó en el último siglo (porque el sector terciario mucho menos dependiente del transporte cobró importancia) (Glaeser \& Kohlhase, 2004), especialmente en países en desarrollo la ubicación periférica de una región es un factor determinante de disparidades espaciales. Mejorar la infraestructura de transporte reduce el tiempo de viaje y de transporte, pero no equilibra desventajas geográficas (Dicken \& Lloyd, 1990: 245).

En la mayoría de las investigaciones citadas, la relación entre las inversiones en infraestructura viaria y el desarrollo económico regional se ha estudiado a nivel nacional, casi siempre tomando el caso de la mejora de la conexión vial entre el centro (económico) y la periferia de un país. En cambio, nuestro artículo explora, fundado en el ejemplo de la carretera chileno-argentina sobre el paso de Jama, la relación entre inversiones en infraestructura de transporte y desarrollo económico local/regional en un contexto transnacional (Figura $\mathrm{N}^{\circ} 1$ ). Porque el paso de Jama es una carretera de alta calidad nuevamente construida en 2005, es muy apto para identificar el efecto de inversiones viarias sobre el desarrollo económico local y regional y a vez conocer los factores que influyen tal desarrollo. El estudio se basa en el análisis de estadísticas tanto oficiales como internas de las aduanas de ambos países, sobre todo con respecto a la evaluación de los existentes flujos de tráfico, de personas y de carga. Para el mejor entendimiento de los resultados de aquel análisis cuantitativo pareció apropiado de usar métodos cualitativos recogiendo las 
Figura $\mathrm{N}^{\circ} 1$

El paso de Jama en los Andes Centrales.

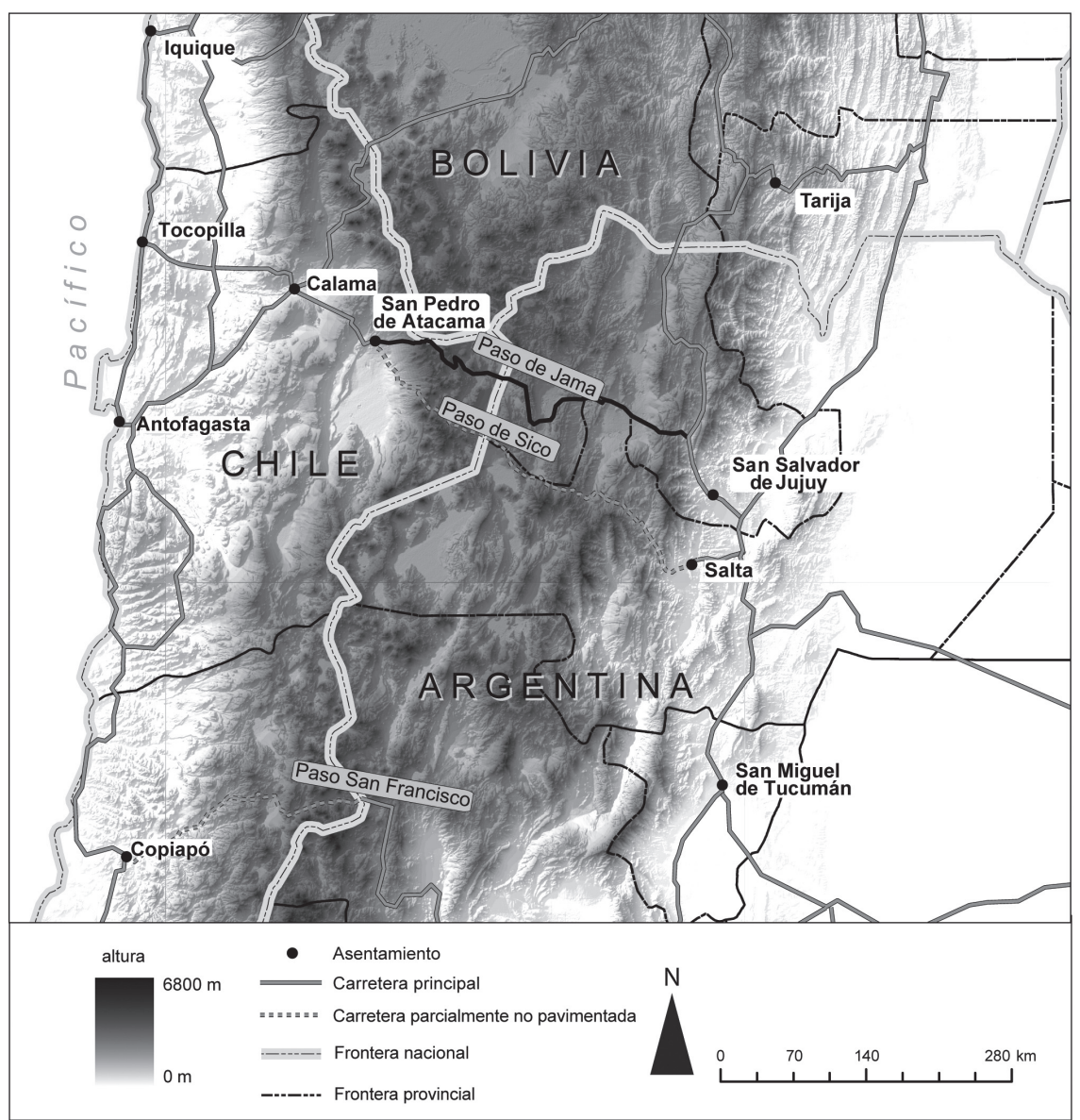

Fuente: Elaboración propia en base a NASA Shuttle Radar Topographic Misión (SRTM) 2000.

informaciones necesarias para identificar los factores influyendo el aprovechamiento del paso de montaña. En 49 entrevistas realizadas en Chile y Argentina con representantes de las cámaras de comercio regionales, empresarios, empleados en la administración pública y colaboradores científicos en varios institutos universitarios de investigación se tematizaron el aprovechamiento actual y las expectativas para el futuro en relación con el eje bioceánico. La selección de los entrevistados se hizo con el fin de obtener una muestra representativa de opiniones de beneficiarios y desfavorecidos (en aspectos económicos, políticos o sociales) de la construcción del paso. Las transcripciones de las entrevistas han sido analizadas y después valuadas considerando la formación profesional, el conocimiento comprobable en cuanto al tema y los intereses personales del entrevistado.

\section{Expectaciones políticas y el potencial económico del paso de Jama}

Una multitud de pasos atraviesa la frontera chileno-argentina, dominado de la cordillera de Los Andes y con más de 4.000 kilómetros de longitud. En 1991, en el marco del "Memorándum de Entendimiento sobre Integración Física", Chile y Argentina se pu- 
sieron de acuerdo de enfocar las inversiones financieras en siete pasos internacionales. Sin embargo, cada región o provincia promociona el desarrollo de "su" paso y encarga opiniones expertas y dictámenes que confirman la necesidad estratégica y el gran beneficio económico del paso favorecido en comparación con pasos fronterizos "competidores"3. El interés creciente en la región andina como punto de contacto para actividades económicas desarrolladas en las regiones subandinas es claramente relacionado con el paradigma neoliberal de ambos países (Amilhat-Szary, 2004).

Tanto Chile como Argentina se caracterizan por sus estructuras centralistas. Tanto el Norte Grande chileno como el Noroeste de Argentina se consideran áreas periféricas, económica y políticamente dependientes de sus capitales respectivas. La minería, especialmente la minería de cobre, convierte la región Antofagasta en un jugador importante en la economía nacional de Chile y un área económicamente floreciente mientras que las provincias del Noroeste de Argentina quedan marcadas por la producción agraria y se ven afectadas de un PBI relativamente bajo (Vázquez Barquero, 2010: 49).

Dada la situación biogeográfica de las dos regiones separadas de la cordillera andina, la idea de un paso de montaña parece sensata: el clima árido del desierto de Atacama no permite más que una producción agraria muy limitada en los oasis, adicionalmente la minería a gran escala absorbe más y más de los pocos recursos hídricos. En las últimas décadas, el crecimiento de la población en el norte de Chile $(+20.3 \%$ entre 1992 y 2002 en la región Antofagasta, comparado con el promedio nacional de $13,2 \%$, INE, 2002) ha incrementado la demanda por productos alimenticios que casi en su totalidad provienen de las zonas agrícolas de las regiones centrales y del sur de Chile.

Al este de la cordillera, el clima de la zona transicional entre el margen trópico y las regiones subtropicales, con precipitaciones advectivas de los vientos alisios, sostiene

3 Ver Gutiérrez (1999) para el paso Pehuenche. una agricultura fructífera que produce más allá de la demanda local y abastece antes de todo el mercado argentino, aceptando en muchos casos una distancia de transporte de más de 1.500 kilómetros. En esta situación, el comercio regional de productos agrícolas a través el paso de Jama parece atractivo para ambas partes: El norte chileno mejora su abastecimiento, el noroeste argentino penetra en nuevos mercados y reduce los costos de transporte a la vez. Adicionalmente, el paso sirve de conexión física entre espacios económicos a escala global, muy precisamente del sur de Brasil, Paraguay, el sur de Bolivia y el norte de Argentina con la zona asiáticopacífica. El objetivo del gobierno argentino de mejorar la integración económica del país en la economía global por medio de reestructurar la economía nacional y regional según el modelo neoliberal incrementó la demanda por una mejor infraestructura de transporte, especialmente para poder intensificar el comercio con los países asiáticos (Schweitzer, 2002: 91).

Se espera que la infraestructura necesaria para el intercambio de mercancías a gran escala, en unión con efectos secundarios, creará empleos a lo largo de la carretera, empleos que se necesitan urgentemente en muchas zonas. Las regiones fronterizas también esperan que el paso promueva el turismo internacional. En ambas partes de la cordillera se encuentran áreas bien conocidas en el mundo turístico: En Chile, es el pueblo San Pedro de Atacama y sus alrededores, en Argentina, la quebrada de Humahuaca es declarado Patrimonio Cultural y Natural de la Humanidad por la Organización de las Naciones Unidas para la Educación, la Ciencia y la Cultura (UNESCO) en 2003. Conectadas por el paso de Jama, ambas zonas ganan atracción en conjunto.

En el período de planificación, los gobiernos de Chile y Argentina justificaron las inversiones enormes necesarias para la construcción del paso con referencia a los impulsos económicos esperados por las regiones (MOP, 1995: parte II). Hasta el día de hoy no se realizó un estudio sobre el uso actual del corredor trasandino. En este trabajo se analiza el tráfico actual sobre el paso, basado tanto en cifras de la aduana chilena (incluyendo el número de vehículos, las mer- 
cancías transportadas, su peso, los puntos de origen y destino de cada vehículo) como en las estadísticas de la Zona Franca de Iquique (ZOFRI).

\section{Cálculo de rentabilidad y cifras del tráfico actual sobre el paso de Jama}

Es esencial examinar las actividades económicas que se conectan por el paso a través de la cordillera andina a dos diferentes escalas espaciales. Las diferencias se encuentran fundamentalmente en el aspecto económico, pero también en el marco político y social: Primero, la Ilamada "integración regional" se refiere (sobre todo) a la interacción económica de las dos zonas fronterizas de Argentina y Chile, es decir el Noroeste Argentina y el Norte Grande. La distancia a superar es pequeña en comparación, dado que tanto origen como destino del tráfico se encuentran en estas zonas. Segundo, se hallan las actividades comerciales que benefician del paso de Jama independiente de su contexto local como etapa de una vía de transporte global. Con este, el paso deviene una ruta de tránsito para el tráfico de carga y de personas entre Brasil y Paraguay a un lado y el espacio circumpacífico al otro.

En el debate sobre la globalización, el regionalismo creciente, y relacionado con aquel la integración regional, se interpreta a veces como una estrategia de adaptación para ajustarse mejor a la economía global. En otras ocasiones está visto como táctica que fortalece la independencia de las regiones en contra del desarrollo global. El "nuevo regionalismo" en América Latina y las diversas proyecciones de integración, no son necesariamente contradictorios a la tendencia global de intensificar el entrelazamiento a nivel mundial. La revitalización (en el caso de los corredores bioceánicos) o el desarrollo de nuevos proyectos de integración regional puede ser sostenido tanto del punto de vista de la economía nacional como bajo el aspecto neoliberal (Kacowicz, 2008: 118).

La mayoría de las mercancías entre AsiaPacífico y Argentina sigue manejado a través del puerto de Buenos Aires. En el aspecto de la logística, la vía de transporte a los importantes centros económicos en el Circumpací- fico es, aún similar en cuanto a la distancia, mucho más fácil cruzando el Atlántico que cruzando el Pacífico porque se ahorra cargar los camiones y el costo del transporte terrestre. Por el momento, el uso del paso de Jama para transportar carga de Asia primordialmente es de interés para Paraguay, aun cuando por la situación de importe semilegal para vehículos usados y electrónica al país es tolerado por el gobierno de Paraguay. Eso significa que la mercancía importada se valora sistemáticamente por debajo de la media con el fin de reducir derechos de aduana.

El análisis de los gastos de transporte de mercancías hacia los importantes puertos asiáticos comparando la vía a través del paso de Jama y Antofagasta por un lado y Buenos Aires y el estrecho de Magallanes o sea el canal de Panamá por el otro, dejó claro que desde el punto de vista económico, la vía de transporte por Jama es atractivo tan solo para transportes que salen cerca del pie de la cordillera, p.ej. en Salta o Jujuy. Sin embargo, la ruta también ahorra tiempo de viaje si el punto de origen se encuentra a más distancia de los Andes. Eso significa que especialmente para mercancía perecedera, la vía de transporte podría ser interesante (Álvarez et al., 1997).

Las estadísticas de la aduana chilena indican que el volumen de carga transportada por el paso de Jama incrementó en los últimos años (Figura $N^{\circ} 2$ ). Salta a la vista que los importes por Jama han aumentado por encima del promedio y en 2005, por primera vez han superado el volumen de exportes. Este desarrollo se debe a los cambios cíclicos de la economía argentina. Inmediatamente después de la "Tango Crisis" de los años 2001/02, la economía se recuperó rápidamente como ya lo había hecho varias veces antes, el PBI creció continuamente de $10 \%$ cada año. Por lo tanto, el tráfico sobre el paso en el marco de la integración regional depende mucho de la situación de las economías nacionales en Argentina y Chile.

La mercancía transportada y las zonas de origen y de destino muestran que el comercio por el paso es atractivo en diferentes perspectivas, por ejemplo para intercambiar productos poco transformados a través de distancias cortas (Ej: del pie de los Andes argentinos 
Figura $\mathrm{N}^{\circ} 2$

Transporte de carga y tráfico sobre el paso de Jama 1997-2010

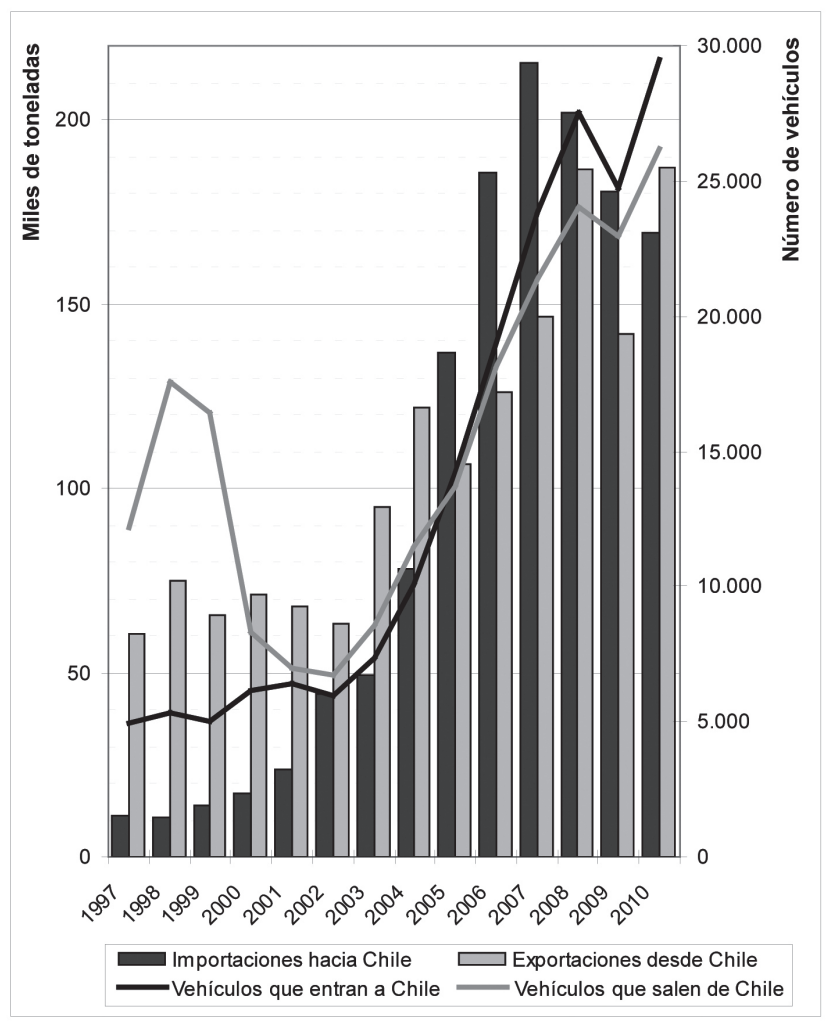

Fuente: Elaboración propia en base a datos de la Aduana de Chile, Síntesis Mensual de Tráfico Fronterizo de Aduanas, 1997-2010.

hacia la costa pacífica), pero también para productos industriales de alta calidad a través de distancias largas (electrónica, partes de vehículos). Otro aspecto del uso actual surge de la situación legal específica de algunos socios comerciales (zona franca, situación de importe en Paraguay). A pesar del crecimiento continuo del volumen de transporte durante los últimos años, la comparación con otros pasos fronterizos entre Chile y Argentina muestra que la importancia general del paso de Jama queda bastante baja (Figura $\mathrm{N}^{\circ} 3$ ).

El análisis de las estadísticas de tráfico aclara que el paso de Jama en absoluto solamente sirve de conexión vial para la integración de las dos regiones fronterizas. Durante el período de investigación, en total $20 \%$ de la carga importada sobre el paso a Chile provenían del Brasil, de Paraguay y Uruguay, en la dirección inversa hasta $47 \%$ de la carga exportada sea enviada a estos tres países (Aduana de Chile). Por lo tanto, gran parte del tráfico sobre el paso es tráfico de tránsito para las regiones fronterizas.

Los políticos, también aquellos de las provincias, promocionaban el paso de Jama desde su inauguración en 2005 como corredor bioceánico, es decir, de enlace vial entre Atlántico y Pacífico. Obviamente, el tráfico de tránsito de los países Brasil, Paraguay y Uruguay es deseado y aumenta la esperanza de un beneficio económico para la región. Sin embargo, preguntado de qué modo los Jujeños por ejemplo se pueden beneficiar del tráfico de tránsito, solamente se responde sin concretar "por infraestructura de servicios" (entrevista con A. Arrueta, Secretario de Planificación y Desarrollo, Municipalidad de 
Figura $\mathrm{N}^{\circ} 3$

Comparación del transporte de carga para los pasos más importantes entre Chile y Argentina 1998-2010.

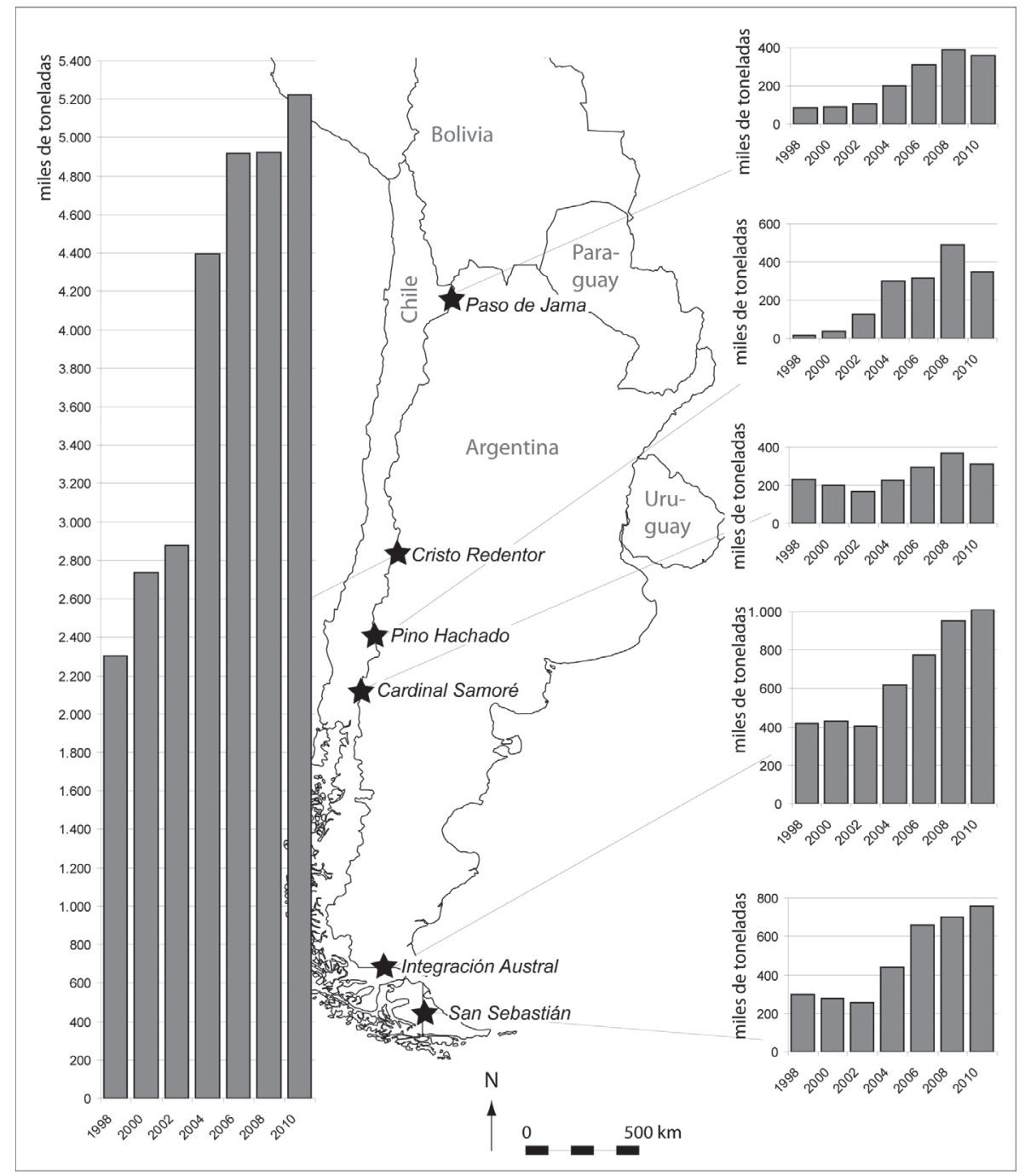

Fuente: Elaboración propia en base a datos de la Aduana de Chile, Síntesis Mensual de Tráfico Fronterizo de Aduanas, 1998-2010.

San Salvador de Jujuy, abril 2006). Mientras que el beneficio del paso de Jama para las regiones fronterizas es evidente bajo el aspecto de la integración regional, porque directamente sacan provecho de la comercialización de sus productos, la ventaja económica del tráfico de tránsito queda dudable.

El sector de transporte, igualmente marcado por la globalización, ya no depende ape- nas de una oferta de infraestructura entre los puntos de salida y de destino y los horarios de la producción just-in-time generalmente no permiten paradas adicionales no necesarias. Algunas gasolineras y talleres a lo largo de la ruta se verán beneficiados, en cambio albergues e infraestructura de abastecimiento aprovecharán en muy menor medida. En Chile, la Región de Antofagasta aprovecha más del tráfico de tránsito a Asia-Pacífico porque gran 
parte de aquel pasa por uno de los puertos chilenos. Del importe y exporte creciente de Brasil, Paraguay y Uruguay a través de Jama los puertos chilenos se benefician mucho más que las provincias argentinas vecinas.

Actualmente no existe una carretera alternativa para el comercio regional entre la provincia de Jujuy y la Región Antofagasta que se caracterizaría por distancia y calidad similar al paso de Jama. No obstante, para el tráfico de personas y carga originado en $y / 0$ destinado a zonas más alejadas el paso de Jama compite con varios pasos vecinos tanto en el sur de Bolivia como en Argentina. Un volumen de tráfico mucho más elevado que el paso de Jama (47.225 vehículos de carga en 2010) tiene el paso chileno-boliviano Chungará (126.364 vehículos de carga, todas las cifras: Aduana de Chile), que ofrece la conexión vial al Pacífico (y a la zona franca de Iquique) no solamente para el tráfico de carga de Bolivia, sino también para Brasil.

Asimismo en segunda la categoría espacial de conexiones globales se encuentran los exportes que salen desde el Noroeste de Argentina a través de Chile hacia países terceros. Aunque aquel tipo de comercio cuenta (junto con la apertura del mercado de consumo en el Norte Grande) entre las grandes esperanzas para el desarrollo económico de las provincias del NOA, el análisis de gastos de transporte aclara que exportar a destinos tan alejados por vía terrestre no es rentable. El Ministerio de Obras Públicas de Chile (MOP 1995: Parte II) ha llegado a la misma conclusión con su modelación de la demanda potencial para el corredor vial sobre el paso de Jama.

\section{Factores del uso de pasos de montaña}

A primera vista, las exigencias económicas para el uso intenso del paso de Jama parecen cumplidas si recordamos la situación económica de las dos zonas fronterizas (véase más arriba). Sin embargo, las estadísticas de la aduana chilena aclaran que aquel paso sigue siendo poco utilizado comparado con otros pasos chileno-argentinos. Las razones que impiden el aprovechamiento de la nueva vía de transporte están discutidas brevemente abajo:

\section{Incertidumbre política}

El motivo más importante para realizar la nueva carretera sobre el paso presumiblemente fue demostrar la buena calidad de las relaciones políticas entre Chile y Argentina (Petit, 2003: 72), pero la confianza de los comerciantes en la estabilidad de aquellas relaciones queda bien limitada. A. León Ruiz, presidente de la Cámara de Comercio en Jujuy, lista la falta de confianza y la ausencia de acuerdos comerciales legalmente obligatorios entre los dos países como los obstáculos más grandes para expandir las relaciones económicas entre ambos lados de la cordillera (entrevista personal, marzo 2006). A pesar de las ceremonias de inauguración bien presentadas y atendidas por los presidentes de ambos países (con ocasión del acabado de la reconstrucción en la parte chilena en 2000 y en la parte argentina en 2005), hasta ahora son pocos los productores y comerciantes que condicionan su existencia económica a la apertura permanente de paso.

\section{Intereses económicos diferentes}

La base de las economías en las áreas en ambos lados de la cordillera andina difiere fundamentalmente. En el mercado mundial, el Norte Grande de Chile con su industria minera puede generar ganancias considerablemente más elevadas que el Noroeste de Argentina con su producción agraria. Esta situación redunda en grandes diferencias del nivel de ingresos. En las regiones de Antofagasta y Tarapacá, las oportunidades de empleos lucrativos generan los salarios medios más altos de Chile mientras que las provincias vecinas de Argentina se cuentan entre las más pobres del país, lo que se manifiesta en salarios bajos. Por los salarios medios elevados, el transporte de los productos agrarios del sur chileno hacia el Norte Grande pueden ser aceptados. Por otro lado, la comparación de la tasa de desempleo en el Norte Grande y el Noroeste argentino alude que la necesidad de encontrar alternativas económicas es bien distinta en las dos zonas: a causa de su 
situación económica, la población argentina es mucho más dependiente de abrir nuevas posibilidades de ganancia que los chilenos; las expectativas respecto al nuevo paso son mucho más grandes en Argentina.

\section{Estructuras de distribución de cadenas de supermercados nacionales}

Durante la última década en Chile, en el comercio al detalle, las cadenas de supermercados nacionales se han impuesto a las estructuras de distribución locales. Esta tendencia es muy perceptible en las grandes ciudades donde los malls recién construidos y bien conectados pueden absorber una gran parte del poder adquisitivo local. Las sucursales de estas cadenas están abastecidas casi en su totalidad por depósitos centrales en su mayoría ubicados en los alrededores del Área Metropolitana de Santiago (AMS) con el fin de simplificar la estructura de distribución para la empresa. Aunque para varios productos, la distancia de transporte es mucho más larga en comparación con productos locales, las oportunidades de ahorro de distancias cortas aparecen económicamente irrelevantes.

\section{Proteccionismo chileno}

Aunque sin duda el interés político apoyó la construcción del paso de montaña, un interés demostrado en diferentes ocasiones y en varias declaraciones (Ballatore, 2005: 293-296), las restricciones de importe de Chile toman rasgos claros de proteccionismo. Por ejemplo, la cuota de azúcar concedida al importe de Argentina a Chile está restringida a un volumen que garantice la venta del azúcar chilena que es considerablemente más cara de producir y transportar. Decisivo en este contexto es el hecho que Chile, a diferencia de Argentina, no es miembro pleno del MERCOSUR (Mercado Común del Sur), sino país asociado y, por lo tanto, no es parte del mercado interior. De esta manera, Chile se reserva la posibilidad de mantener restricciones aduaneras o contingentes de importe.

\section{Reglamentos fitosanitarios}

Para el comercio de productos agrícolas, los reglamentos fitosanitarios del SAG chile- no (Servicio Agrícola y Ganadero) significan un obstáculo considerable. Con el objetivo de mantener el país libre de enfermedades animales y vegetales y por consecuencia también sus productos de exporte, para cada país de origen Chile ha establecido normas exactas que reglamentan para cualquier producto agrícola y ganadero las posibilidades y modalidades exactas de importe. En el caso de Argentina, por las enfermedades existentes en este país los reglamentos se han formulado tan estrictos que la mayoría de los productos no puede ser importada sin procesamiento industrial o un tratamiento especial que es caro para los productores y asociado con una burocracia notable.

\section{Tradiciones étnicas e históricas}

La población autóctona de la zona fronteriza chilena, los atacameños, tradicionalmente no es una comunidad que se gana la vida con el comercio. Incluso el turismo internacional floreciente que se desarrolla en San Pedro de Atacama y sus alrededores se impulsa por factores exógenos. La comunidad atacameña no aprovecha las nuevas oportunidades que ofrece el paso, sobre todo para las actividades turísticas, porque (según informaciones propias) carecen de experiencia y de formación en el sector turístico. En la parte argentina de la frontera, la población endógena tiene la tendencia de considerar la Puna como recurso amenazado por la carretera construida (Petit, 2003: 74).

Muy distinto a eso se comporta la población aimara que se origina en la zona fronteriza boliviana cercana: con mucha habilidad y éxito los aimara crean empresas tanto turísticas como comerciales, y con ello continúan su tradición histórica de comerciantes.

\section{Restricciones legales}

Los comerciantes que importan o exportan sobre el paso de Jama se quejan sobre todo de obstáculos burocráticos al paso de frontera chileno-argentina. No fue antes de 2009 que se inauguró un complejo fronterizo común cerca del paso para reducir el tiempo de espera al cumplir las formalidades aduaneras. Otros impedimentos legales siguen 
existiendo: la prohibición mutua de ofrecer viajes turísticos transfronterizos constituye una restricción considerable para el turismo. Los tour operadores bolivianos se han adaptado a estas reglamentaciones de manera que ofrecen los viajes transfronterizos oficialmente con dos agencias de viaje (una situada en Chile y otra en Bolivia) y simplemente cambian de vehículo en la frontera chilenoboliviana. Entre Argentina y Chile, todavía no existen actividades transfronterizas comparables.

Como las empresas de alquiler de ambos países no permiten cruzar la frontera nacional con sus vehículos, a los turistas solo les queda como opción utilizar el automóvil propio o el transporte público para viajar sobre el paso de Jama al país vecino. Durante los meses de vacaciones en verano, las empresas chilenas de autobús que ofrecen el viaje sobre el paso no logran satisfacer la demanda.

\section{Conclusiones}

A primera vista puede parecer que las condiciones señaladas por Banister y Berechman (2001: 210) como esenciales para generar un impulso económico de la inversión en infraestructura de transporte se han cumplido en el caso de Jama. El marco económico y las condiciones para inversiones pueden ser favorables. Sin embargo, el apoyo político e institucional se restringe más bien a palabras por parte de los gobiernos nacionales y los reglamentos y restricciones legales siguen siendo los obstáculos más grandes al crecimiento del comercio. Al mirarlo en detalle, hemos visto que la situación a los dos lados de la frontera chileno-argentina son bien diferentes y reconocimos los factores de influencia para el uso y no uso del paso.

Al condensar los factores identificados del estudio de caso en un esquema más general (Figura $N^{\circ} 4$ ), se llega a la conclusión bajo qué condiciones la inversión en infraestructura de transporte en una región resultará en un desarrollo económico endógeno: 1 . el potencial económico, es decir la rentabilidad del transporte de personas y carga; 2. el marco político/ legal, es decir el apoyo y las restricciones al sector de transporte; 3 . las condiciones de infraestructura, es decir servicios de infraestructura o la red de transporte ya existente; 4. el marco étnico/ histórico, es decir las tradiciones económicas de la población local. Solo si las cuatro condiciones son favorables a la vez, la región podrá desarrollarse económicamente por actores endógenos. Asimismo, el esquema muestra los cambios alternativos para la región si falta uno de los factores esenciales para el desarrollo endógeno. Verbigracia, la carencia de apoyo político y legal (por ejemplo, restricciones aduaneras) puede resultar en actividades ilegales (contrabando), si el potencial económico, las condiciones de infraestructura y las tradiciones étnicas e históricas son idóneas para las actividades económicas. Pero el esquema permite no solo el análisis del significado económico de infraestructura ya existente y la identificación de factores que impiden el desarrollo económico endógeno. Con la figura también es posible comparar una ruta proyectada con rutas alternativas evaluando los cuatro factores de cada una. Tal análisis puede identificar las variables que limitan el desarrollo económico y abrir el debate político de adaptar los factores impedientes al desarrollo.

Mientras que Banister y Berechman (2001: 210) en sus reflexiones no hacen caso de las influencias étnicas e históricas, nuestro estudio de caso mostró la importancia que pueden tener para la integración de la población local en las actividades comerciales y/o turísticas. Y finalmente, este factor es aquel que menos se puede adaptar por alguna decisión política. Los autores tampoco otorgan mucha importancia a las variabilidades en la persistencia de los factores aunque la perduración de estructuras es una variable esencial para saber en qué período se pueden esperar cambios.

El desarrollo en el caso del paso en los próximos años bajo aspectos económicos y sociales dependerá de cómo cambiarán los factores listados arriba. La Figura $N^{\circ} 4$ indica las grandes diferencias en la persistencia temporal de cada uno de los factores. Mientras que el marco jurídico de restricciones de importe y obstáculos burocráticos puede ser adaptado en poco tiempo, factores como las tradiciones económicas de un grupo étnico quedan estables en períodos bastante largos. 
Figura $N^{\circ} 4$

Factores esenciales para el estímulo del desarrollo económico regional por inversión en infraestructura de transporte

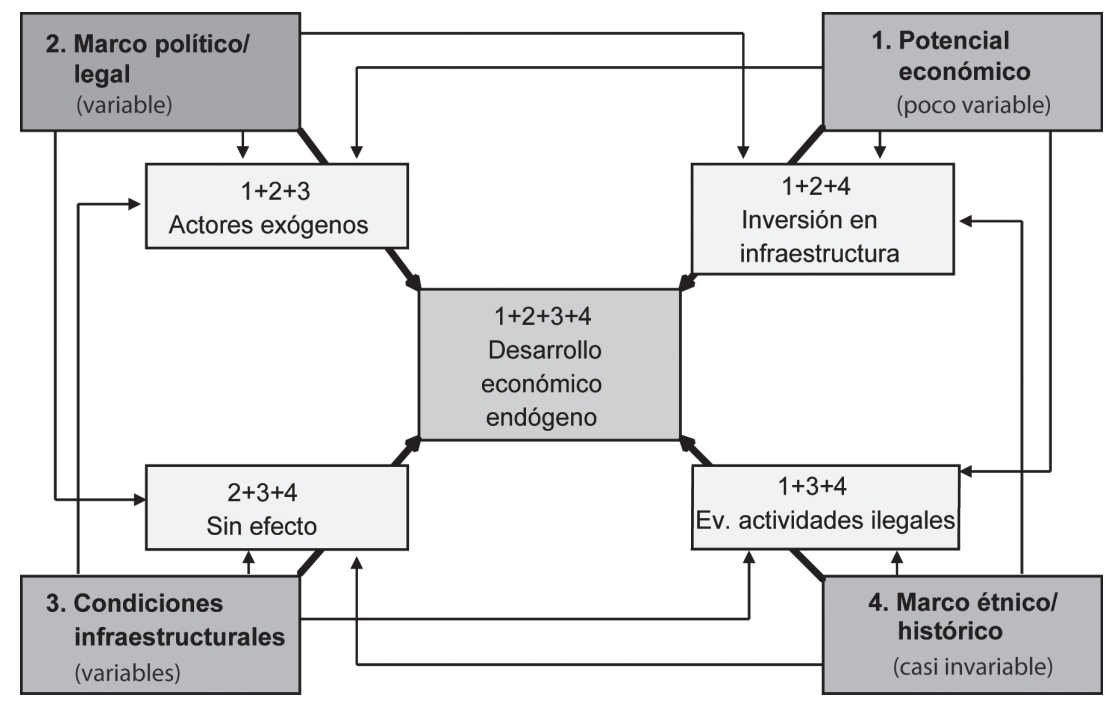

Fuente: Elaboración propia.

\section{Referencias bibliográficas}

ADUANA DE CHILE. Serie Mensual de Tráfico terrestre 1997-2010. Disponible en Internet: http://www.aduana.cl/prontus_aduana/ site/artic/20070418/pags/20070418084613. html.

ÁlVAREZ, F.; AROCA, P.; CLAPS, D. y LUFIN, M. Estudio de Tarifas de Transporte de Mercaderías de la Zona del Centro Oeste Sudamericano a los Mercados Internacionales. In: CASTELLO, I.R.; KOCH, M.R.; OLIVEIRA, N.; SCHAFFER, N.O. e STROHAECKER, T.M. (editores). Fronteiras na America Latina, espaços em transformaçao. Seminario internacional: As Areas de Fronteira da América Latina no Novo Patamar da Economia Capitalista. Porto Alegre: Editora da UniversidadeUFRGS/FEE, RS, 1997, p. 265-279.

AMILHAT-SZARY, A.-L. Les nouvelles traversées andines. De la montagne-obstacle á la montagne-interface? Cahiers de Géographie, 2004, № 2, p. 121-128.
BALLATORE, I. Jama. La causa que movió montañas. San Salvador de Jujuy: Ediciones PGA, 2005.

BANISTER, D. \& BERECHMAN, Y. Transport investment and the promotion of economic growth. Journal of Transport Geography, 2001, No 9, p. 209-218.

BORSDORF, A. Verkehrs- und Städtenetze in Alpen und Anden. Über die Problematik der Übertragbarkeit von Erfahrungen im internationalen Entwicklungsdialog. 54. In: GAMERITH W.; MESSERLI, P. y MEUSBURGER, P. (Hg.). Alpenwelt - Gebirgswelten. Inseln, Brücken, Grenzen. Tagungsbericht und wissenschaftliche Abhandlungen. Heidelberg: DGfG, 2004, p. 299-308.

BUTTON, K. Transport Economics. Cheltenham: Elgar, 2010.

DICKEN, P. \& LLOYD, P. Location in Space. Theoretical Perspectives in Economic Geography. New York: HarperCollins, 1990. 
ECKEY, H.-F. \& KOSFELD, R. New economic geography. Critical reflections, regional policy implications and further developments. Kassel: Universidad de Kassel, 2004.

GLAESER, E. \& KOHLHASE, J. Cities, regions and the decline of transport costs. Papers in Regional Science, 2004, Vol. 1, No 83, p. 197-228.

GutiérreZ, G.E. Paso del Pehuenche: Una vía de integración entre el MERCOSUR y Chile. Revista Universum, 1999, No 14, p. 81-90.

INSTITUTO NACIONAL DE ESTADÍSTICAS (INE). XVII Censo de población y VI de vivienda. Santiago de Chile: INE, 2002.

KACOWICZ, A. América Latina en el mundo: globalización, regionalización y fragmentación. Nueva Sociedad, 2008, № 214, p. 112-123.

KRUGMANN, P. Geography and Trade. Leuven: Leuven University Press, 1991.

LAKSHAMANAN, T.R. \& CHATTERJEE, L.R. Economic consequences of transport improvements. Access, 2005, No 26, p. 28-33.

MACKINNON, D.; PIRIE, G. \& GATHER, M. Transport and Economic Development. In: KNOWLES, R., SHAW, J. \& DOCHERTY, I. (editors). Transport Geographies. Mobilities, Flows and Spaces. Oxford: Blackwell, 2008, p. 10-28.

MINISTERIO DE OBRAS PÚBLICAS (MOP). Informe Final. Seminario "Potencialidades de Integración en América Latina, Corredores Bioceánicos: Expectativas y realidades". Santiago de Chile: CEPAL, 1995.
NJENGA, P. \& DAVIS, A. Drawing the road map to rural poverty reduction. Transport Reviews, 2003, Vol. 2, No 23, p. 217 241.

OLSSON, J. Improved road accessibility and indirect development effects: evidence from rural Philippines. Journal of Transport Geography, 2009, Vol. 6, No 17, p. 476-483.

PETIT, J. Les territoires de passage à I'épreuve des aménagements transfrontaliers dans les Andes de la Puna. Revue de Géographie Alpine, 2003, Vol. 3, No 91, p. 71-82.

SALLING, K.B. \& BANISTER, D. Assessment of large transport infrastructure projects: The CBA-DK model. Transportation Research Part A: Policy and Practice, Vol. 9/10, 2009, No 43, p. 800-813. Disponible en internet: http://dx.doi.org/10.1016/j. tra.2009.08.001

SCHWEITZER, M. El sistema de transporte en el Cono Sur: Los nuevos proyectos. Estudios Fronterizos, 2002, Vol. 6, No 3, p. 89-121.

VÁZQUEZ BARQUERO, A. Diversidad territorial y desarrollo endógeno en Argentina. Revista Cultura Económica, 2010, № 77/78, p. 46-72.

VICKERMAN, R.W. (editor). Infrastructure and regional development. European Congress of the Regional Science Association. Istanbul 1990. London: Pion, 1991.

WILSON, G.W. Towards a theory of transport and development. In: HOYLE, B.S. (editor). Transport and Development. London: Macmillan, 2009, p. 208-230. 